\title{
An Undiagnosed Non Functioning Retroperitoneum Paraganglioma, Complicating A Gynaecological Surgery; A Case Report
}

Noraziana AW ${ }^{\mathrm{a}}$, Hakim B ${ }^{\mathrm{a}}$, Alik $\mathrm{R}^{\mathrm{b}}$ and Mokhtar $\mathrm{A}^{\mathrm{a}}$

aDepartment of Obstetrics and Gynaecology, Faculty of Medicine, International Islamic University Malaysia, Kuantan, Pahang, Malaysia

${ }^{b}$ Department of Obstetric and Gynaecology, Hospital Tengku Ampuan Afzan, Kuantan, Pahang, Malaysia.

ABSTRACT

Paragangliomas are uncommon tumors arising from the neuroendocrine elements (chief cells) of the paraganglia and symmetrically distributed along the aorta in close association with the sympathetic chain. Although functional tumors are easier to diagnose due to the clinical findings caused by excess secretion of catecholamines and an elevation of the urinary catecholamine levels, however the diagnosis of nonfunctioning paraganglioma of the retroperitoneum is difficult and only few cases have been made before exploratory laparotomy. Although our patient was initially planned and operated by our gynaecological team, the incidental findings had to involve the general surgeons. She is fortunate that the tumour did not show any features of malignancy nor metastasis, however a long time follow up is needed in case of any possible recurrence.

KEYWORDS: Paraganglioma, Non-functioning, Retroperitonium

\section{INTRODUCTION}

Paragangliomas are uncommon tumors arising from the neuroendocrine elements (chief cells) of the paraganglia and symmetrically distributed along the aorta in close association with the sympathetic chain. This tissue either accumulates in the adrenal medulla, where it gives rise to intra-adrenal paraganglioma, also known as pheochromocytoma, or remains in its paraaortic sites, where it may develop into extra-adrenal, retroperitoneal paragangliomas. 1 Males are affected more frequently than females and most patients are diagnosed between 30-45 years of age. Most tumours are hormonally active (functional) and cause clinical findings associated with the excess secretion of catecholamine. These include headaches, sweating, palpitation and hypertension. The most common origin for extra-adrenal retroperitoneal paragangliomas is the paraganglions between the inferior mesenteric artery and the aortic bifurcation, which are known as the organs of Zuckerkandl.

\section{CASE REPORT}

A 54 years old para 3 presented with a history of menorrhagia for the past six months. She has a his-

Corresponding author;

Dr Hakim Gharib Bilal

Department of Obstetric and Gynaecology

International Islamic University Malaysia

25200 Kuantan, Pahang, Malaysia.

e-mail: hg_bilal@yahoo.com tory of partial thyroidectomy which was done 15 years ago, currently she is under surgical follow up for recurrent toxic multinodular goitre. Because of her current treatment with carbimazole $10 \mathrm{mg}$ two times a day she is asymptomatic of palpitation, heat intolerance and there was no climacteric symptoms. She did not have a history of hypertention or diabetic mellitus.

Obstetric history revealed a three previous caesarean section and bilateral tubal ligation. On examination she found to be pale with a $3 \times 3 \mathrm{~cm}$ thyroid swelling, the pulse and blood pressure were normal. Abdominal examination revealed an 18 weeks size uterus with ultrasonic features of fibroid, a normal findings for hysteroscopy and diagnostic curettage and the histological results showed a secretory endometrium. Pelvic and abdominal CT Scan was not done. She was counselled and booked for total abdominal hysterectomy and bilateral sulpingoophorectomy (TAHBSO) on 23rd March 2009.

At laparotomy the uterine fibroid was noted and a standard TAHBSO procedure was performed. Incidentaly another tumour was noted at the abdominal aorta just before the bifurcation of common iliac arteries, during manipulation of the tumour the blood pressure shoot up to $230 / 130$ mmhg with pulse rate of 120 beats per minutes. She was given intravenous (IV) labetolol and IV esmolol (2 times).Considerable dissection was required to remove the tumour from the vessels, the tumour with its marginnwas succesfully removed measuring $5 \mathrm{~cm}$ by $5 \mathrm{~cm}$ (Figure 1). No intraabdominal metastases were observed.

After the removal of the tumour the blood pressure suddently dropped and because she did not respond 
to ephedrine and phenylephrine, she was started on intravenous dopamine and post operatively she was admitted in intensive care unit (ICU) for closed observation. The estimated blood loss was $1000 \mathrm{ml}$ and two units of packed cell was transfused post operatively. She was given standard antibiotic prophylaxis and subcuticular heparin as thromboprophylaxis and blood pressure monitoring. She was transfered to gynaecological ward the following day with an uneventful recovery before allowed home on the fith day post operation to be followed up in clinic.

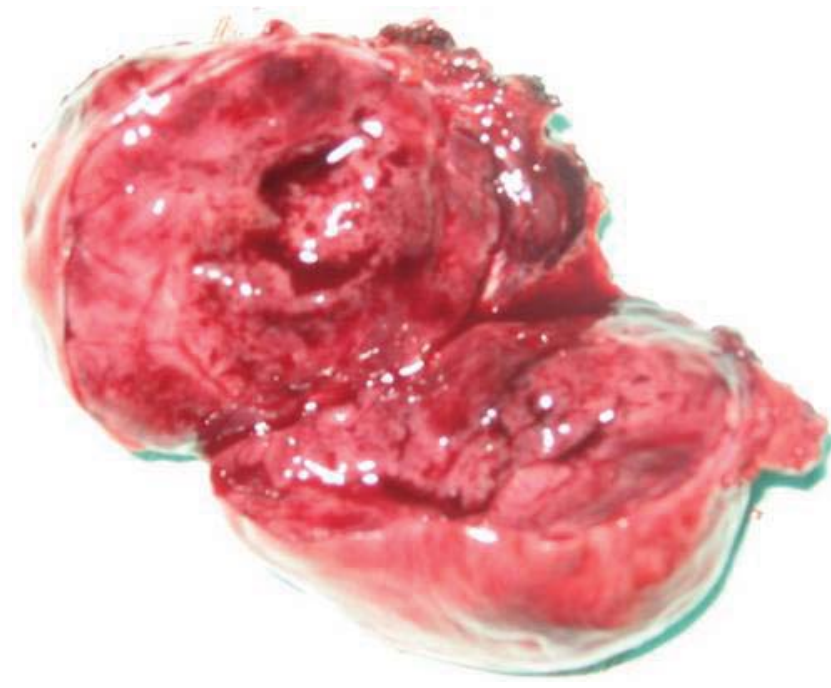

Figure 1. Gross specimen showing a cut section which shows a firm greyish surface, no haemorrhagic areas seen

\section{PATHOLOGICAL REPORT}

The specimen consist of an oval encapsulated greyish tissue measuring $50 \times 35 \times 30 \mathrm{~mm}$. The tumour was already cut to show firm greyish surface. No haemorrhagic area seen grossly. A complete cut sections submitted in two blocks.. Section of the well circumscribed tumour shows to be composed of well-defined nests of cuboidal cells seperated by highly vascularised fibrous septa.. The nuclei was centrally placed with granular cytoplasm.

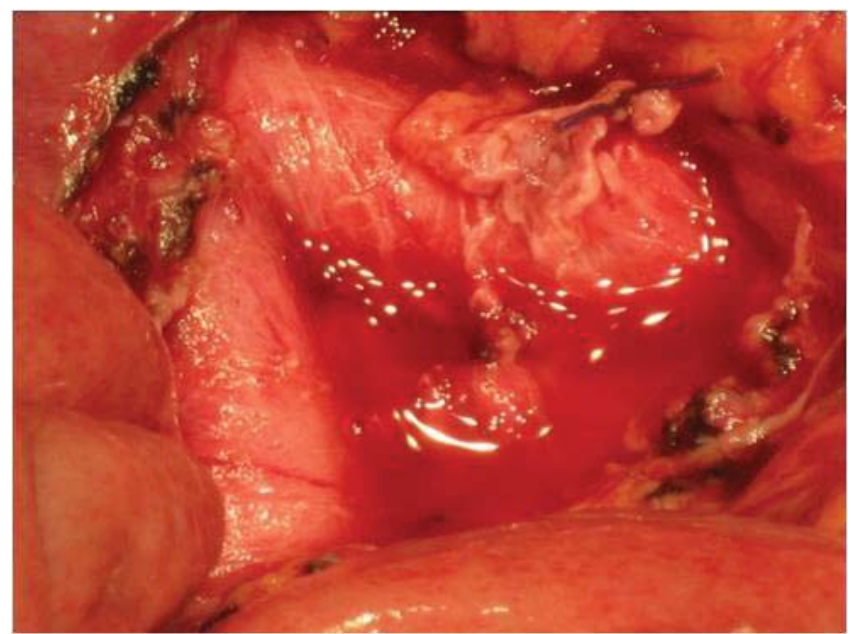

Figure 2. At the bifurcation of the aorta, a complete ressection of the tumour was ensured
Immunohistochemistry:

Cytokeratin - negative

Chromogranin - positive

Neurospecific enolase - positive

Synaptophyrin - negative

Epithelial membrane antigen - negative

S- 100 - inconclusive

Interpretation: Paraganglioma

The uterine specimen shows a uterine fibroid and both ovaries have endometriotic cyst.

\section{DISCUSSION}

Paragangliomas have been reported on multiple locations. Retroperitoneal paragangliomas are divided into adrenal, whereby $10 \%$ have distant metastases at diagnosis and approximately $25-60 \%$ are functional, with symptoms and signs of norepinephrine overproduction compared with the adrenal paraganglioma (pheochromocytoma) in which there may be overproduction of epinephrine and norepinephrine. ${ }^{2}$ The exact incidence of extra-adrenal retroperitoneal paraganglioma is not known. Diagnosis of retroperitoneal paraganglioma is infrequently made unless the tumour is functional. ${ }^{3}$ Our patient who had been asymptomatic with bilateral endometrioma and a large uterus with uterine fibroid and a history of three previous caessarean section, it was not easy to diagnose retroperitoneum paraganglioma preoperatively. Functional tumors are easier to diagnose due to the clinical findings caused by excess secretion of catecholamines and an elevation of the urinary catecholamine levels. ${ }^{4}$ In nonfunctional extra-adrenal retroperitoneal paragangliomas, diagnosis usually depends on nonspecific factors related to the growth of a retroperitoneal mass or occasionally showing incidental symptoms of metastases, which could be seen up to 7 years after resection. ${ }^{5}$ For this reason, patients should continue to be followed carefully for recurrence.

Previous reports in the literature reveal a malignancy rate of $22 \%-50 \%{ }^{3}$ By comparison, the rate of malignancy is about $10 \%$ for paragangliomas of the adrenal gland. Malignant tumors tend to be large, necrotic, and poorly marginated. However, these same features maybe found in tumors that are not malignant, and therefore they cannot be used as predictors to differentiate between benign or malignant nature of the tumor. ${ }^{6}$ The criteria that are applicable to other tumors such as cellular atypia, necrosis, vascular invasion, and mitotic activity cannot be used to define malignancy and are therefore of no use for an assessment of prognosis. ${ }^{6}$ The only reliable indicators of malignancy are an extensive invasion of the surrounding organs and remote metastatic spread.

Once the diagnosis of retroperitoneal paraganglioma is made, every attempt should be made to perform a complete surgical resection. These tumors progress slowly, and patients have a fairly good survival rate even when they recur locally and develop remote metastases. $^{7}$ 


\section{CONCLUSION}

The diagnosis of nonfunctioning paraganglioma of the retroperitoneum is difficult and only few cases have been made before exploratory laparotomy. Although our patient was initially planned and operated by our gynaecological team, the incidental findings had to involve the general surgeons. She is fortunate that the tumour did not show any features of malignancy nor metastasis, however she needs a long time follow up for any possible recurrence.

\section{REFERENCES}

1. Hayes WS, Davidson AJ, Grimley PM, Hartman DS. Extraadrenal retroperitoneal paraganglioma: clinical, pathologic, and CT findings. Am J Roentgenol 1990;155:1247-50

2. Enzinger FM, Weiss SW. Liposarcoma, soft-tissue tumors. 2nd edn. Washington: Mosby, 1988: 83660

3. Sclafani LM, Woodruff JM, Brennan MF. Extraadrenal retroperitoneal paragangliomas: natural history and response to treatment. Surgery 1990; 108:1124-1129

4. Samaan NA, Hickey RC. Pheochromocytoma. Semin Oncol 1987; 14:297-305

5. Law NW, Alfano L. Non-functioning retroperitoneal paraganglioma. J R Soc Med 1987; 80:246-7

6. Glenn F, Gray GF. Functional tumors of the organ of Zuckerkandl. Ann Surg 1976; 183:578-86

7. Montresor E, lacono C, Nifosi F, et al. Retroperitoneal paragangliomas: role of immunohistochemistry in the diagnosis of malignancy and in assessment ofprognosis. Eur J Surg 1994; 160:547-52 
